\title{
Verletzungen des Akromio- und sternoklavikulären Gelenkes
}

\author{
Dara Orangi, Karl Heinrich Winker
}

\section{Zusammenfassung}

Das immer noch kontrovers diskutierte Problem der Therapie der Schultereckgelenksverletzung wurde bereits vor 40 Jahren von J. D. Tossy mit folgendem Satz beschrieben: „Das Dilemma besteht nicht in der Auswahl der Operationsmethode, sondern darin, welche Verletzungsform einer chirurgischen Therapie bedarf.“ Über die konservative Behandlung der Tossy-I-bzw. Rockwood-I-Verletzung besteht Konsens. In der grundsätzlichen Entscheidung der Therapieform bei instabilen Verletzungstypen besteht keine Einigkeit. Die Vielzahl der angewandten Operationsmethoden und die bunte Auswahl der Implantate zeigen die Unstimmigkeit in der Frage des geeigneten Verfahrens. Die Beurteilung der Behandlungsergebnisse in den wenigen prospektiv randomisierten Untersuchungen zeigt

\section{Aufbau und Funktion}

Zur Sicherung der Mobilität des Armes besteht eine bewegliche, stabile Verbindung des Schlüsselbeines mit dem Brustbein einerseits und dem Schulterblatt andererseits. Diese dynamische Fixierung ist Voraussetzung für die außergewöhnliche Bewegungsfreiheit im Schultergelenk.

Das körpernahe Ende der Klavikula geht mit dem Manubrium sterni eine knöchern sehr instabile Gelenkverbindung

OP-JOURNAL 2002; 18: 200-207

(C) Georg Thieme Verlag Stuttgart · New York vergleichbar gute Ergebnisse der konservativen und operativen Behandlung der ACG-Verletzung höheren Grades. Als operative Therapiemethode wurde im Jahre 2001 in einer Umfrage an unfallchirurgischen Kliniken in Deutschland von 37\% der 104 Befragten die temporäre Transfixation des Schultergelenkes mit KirschnerDrähten angegeben. 32\% der Befragten wählten die korakoklavikuläre Cerclage als Methode der Wahl. Die seltene Verletzung des sternoklavikulären Gelenkes wird nach Allmann in 3 Schweregrade unterteilt. Die instabile Typ-III-Verletzung wird je nach Luxationsrichtung der Klavikula in eine vordere und eine hintere Luxation eingeteilt. Die Behandlung der Luxation besteht in der Reposition und Stabilisierung mittels Drahtcerclage oder einer Platte. Die Typ-I-oder Typ-II-Verletzung des sternoklavikulären Gelenkes wird konservativ behandelt.
Das Sternoklavikularband verhindert die vordere und hintere Instabilität. Das Kostoklavikularband, zwischen Schlüsselbein und 1. Rippe, verhindert eine kraniale Dislokation (Abb.1).

Das Gelenk weist eine relativ große Bewegungsfreiheit in Rotation (bis $\mathrm{zu} 40$ Grad) und Elevation auf.

Das Schultereckgelenk (Akromioklavikulargelenk, ACG) besteht aus einem Kugelgelenk und einer Syndesmose.

Das Kugelgelenk wird aus dem Akromion und der Klavikula gebildet, dessen Bewegungen in 3 Ebenen stattfinden. Der höchste Bewegungsumfang wird in der axialen Rotationsfreiheit mit bis zu 4550 Grad angegeben. Diese Rotationsbewegung findet vor allem bei der Abduktion bzw. Elevation des Armes und zwar unterhalb von 30 Grad und oberhalb von 135 Grad statt.

Das Gelenk besitzt einen Discus articularis, eine Gelenkspaltweite von 5-7 mm wird als normal angegeben, der Diskus ist nach Angaben einiger Autoren nicht immer angelegt.

ein, das Sternoklavikulargelenk, SCG. Es handelt sich um ein Kugelgelenk, welches durch das 2-teilige Sternoklavikularband, das Ligamentum interclaviculare und einen intraartikulären Diskus verstärkt wird.
Das Akromioklavikularband besteht aus einem oberen und einem unteren Anteil, wobei die Pars superior den stärkeren Anteil darstellt, diese Bandverbindung, die hauptsächlich die horizontale Stabili-

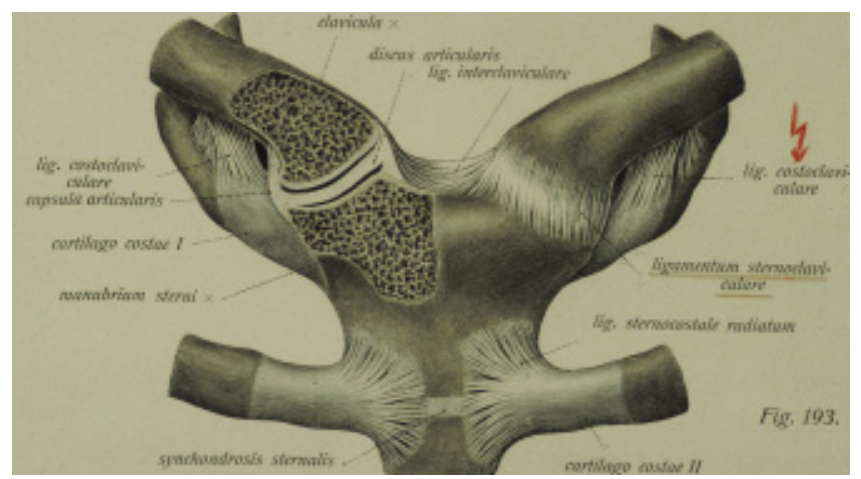

Abb.1 Aufbau des SCG. 

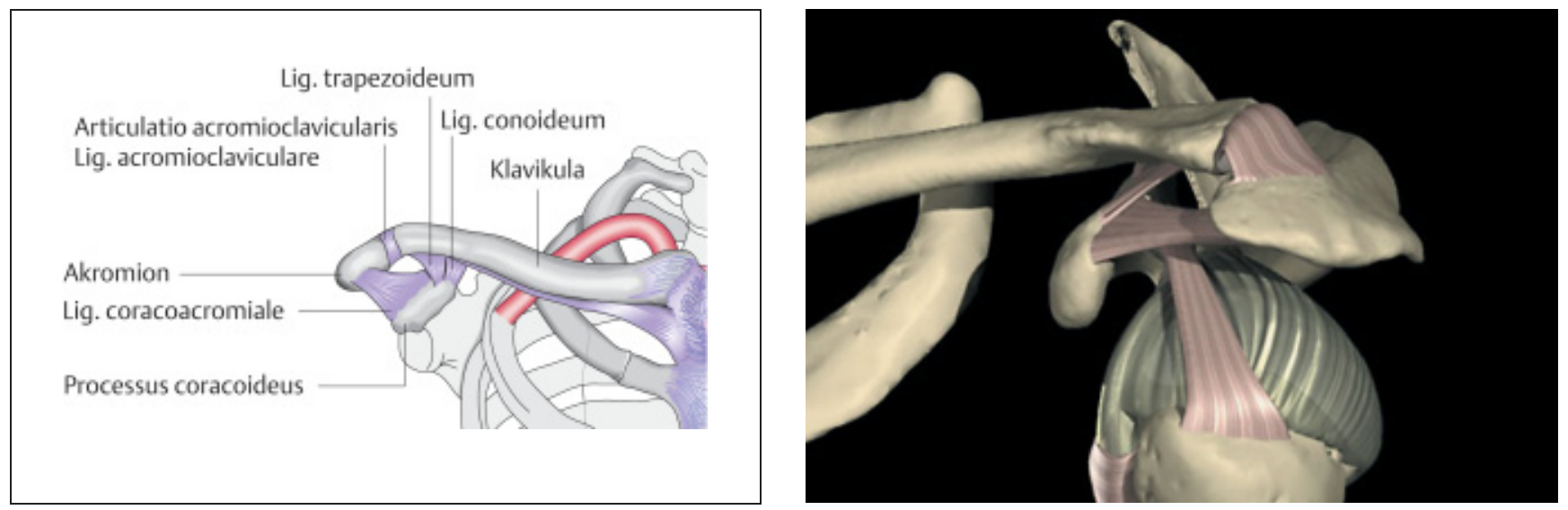

Abb. 2 u. 3 Bandverbindung des ACG.

tät sichert, besitzt eine Reißfestigkeit von ca. $36-40 \mathrm{kp}$.

An der Klavikula sowie am Akromion setzen die Aponeurosen des Deltamuskels und des M. trapezius als zusätzliche Stabilisatoren an.

Die Syndesmose besteht aus 2 Bändern (der medialen Pars coronoidea und der lateralen Pars trapezoidea). Sie stellt die funktionelle Einheit der korakoklavikulären Bandverbindung dar und erhält die durchschnittlich mit $1,3 \mathrm{~cm}$ angegebene Distanz zwischen Korakoid und Klavikula (Abb. 2 u. 3).

In Leichenpräparaten zeigten korakoklavikuläre Bänder eine Reißfestigkeit von $80 \mathrm{kp}$ und mehr, ihre Aufgabe ist die Sicherung der vertikalen Stabilität $[1,9,13,14,16]$.

\section{Unfallmechanismus}

Die sternoklavikuläre Luxation ist eine sehr seltene Verletzung. Rowe berichtet über 34 Dislokationen dieses Gelenks innerhalb von 40 Jahren (1958-1998), unter 1603 Schulterverletzungen bis zum Jahre 1958 fand er 13-mal Luxationen des SCG. Die meisten Verletzungen sind ventrale Luxationen. Ursache dieser Ver- letzung ist häufig eine indirekte Gewalteinwirkung. Die posteriore Luxation ist eine Rarität und wurde erstmals von Rodrigues im Jahre 1843 beschrieben. Eine direkte Gewalteinwirkung auf die mediale Klavikula führt zu diesem Luxationstyp $[6,14]$.

Die Inzidenz der ACG-Luxation liegt bei ca. 3-4\% aller Gelenksluxationen. Es sind überwiegend Männer betroffen, meistens handelt es sich um Verkehrsund Arbeitsunfälle. Bei diesen Unfällen liegt der Altersdurchschnitt zwischen 35 und 40 Jahren. Bei Sportunfällen liegt der Altersdurchschnitt bei 25 Jahren. Das direkte Trauma mit dem Sturz auf die Schulter zeigt sich bei Fußballspielern (daher der Name Football Shoulder) oder bei Ringern, Boxern, Judokas, Reitern, Radfahrern und Gewichthebern (Abb. 4 bis 6). In der Statistik der Sportverletzungen der Schulterregion nehmen Schultereckgelenksverletzungen den 3 . Platz, die Sternoklavikulargelenk-Luxationen den 4. Platz ein $[1,3,6,8]$.

Die zur Sprengung des Schultergelenkes führenden Kräfte wirken von oben auf das Arkomion ein und sind abwärts, leicht nach vorne und medial gerichtet, dies führt zur Verlagerung des Schulterblattes nach unten und medial. Solange
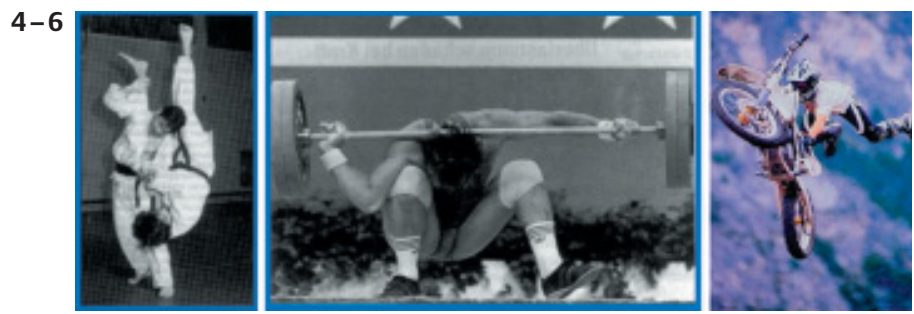

Abb. 4 bis 6 Sturz auf die Schulter, ACG-Sprengung infolge der abwärtsgerichteten Krafteinwirkung auf das Acromion bei Radsport- oder Kraftsportarten. Von oben auf das Acromion einwirkende Kraft wie beim Gewichtheben.

die korakoklavikulären Bänder intaktbleiben, kommt es zu keiner vertikalen Instabilität. Die isolierte Durchtrennung der akromioklavikulären Bänder verursacht lediglich eine Subluxation des Gelenkes.

\section{Diagnostik}

Der Inspektion und Palpation kommen eine besondere Bedeutung zu, die Diagnose einer SCG- oder ACG-Sprengung ist eine klinische Diagnose (Abb.7-12).

Schwellung und Druckschmerz weisen auf Vorliegen einer SCG-Luxation bzw.

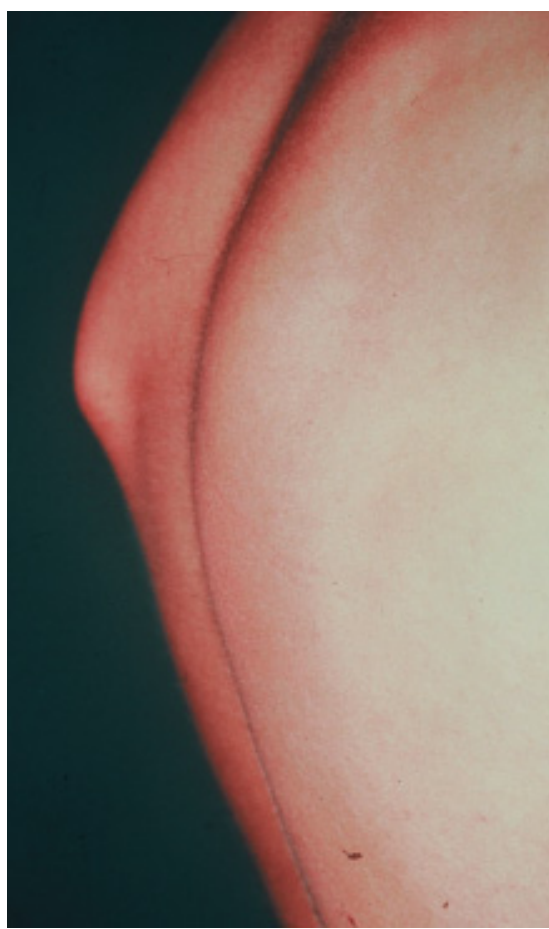

Abb.7 Das klinische Bild einer chronischen SC-Luxation. 

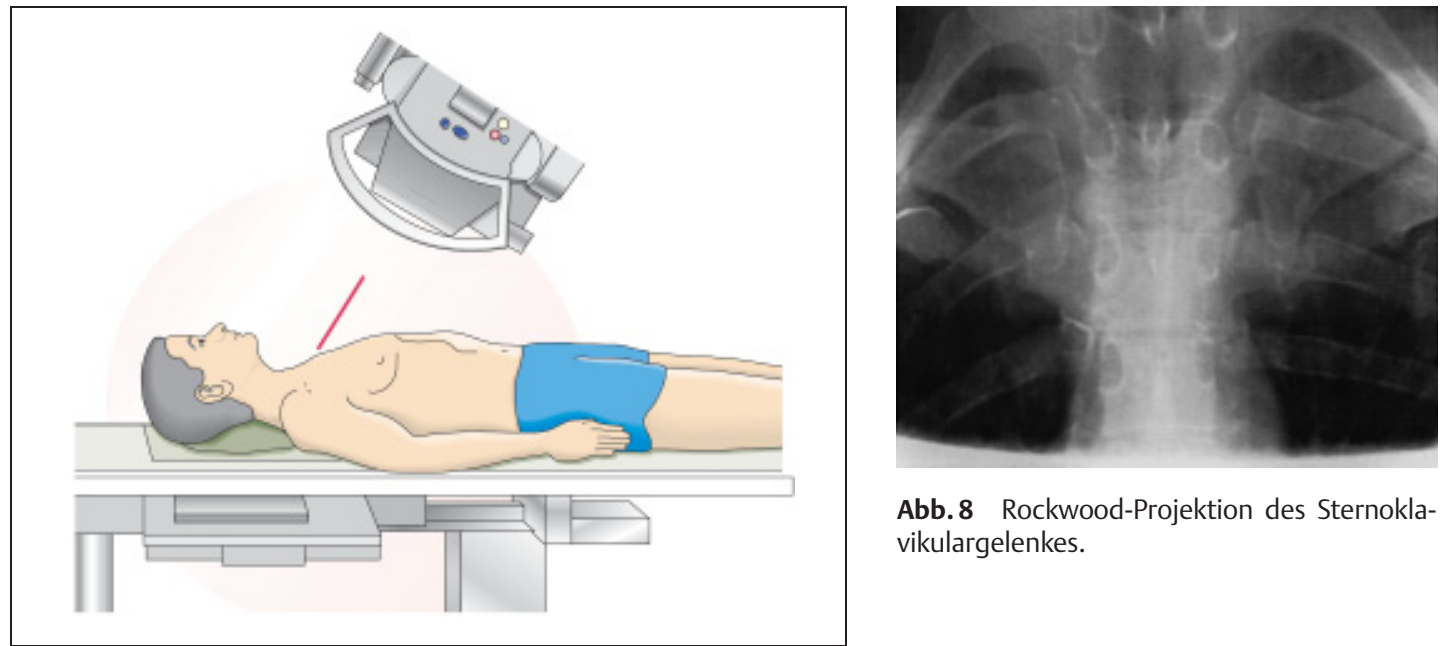

Abb. 8 Rockwood-Projektion des Sternoklavikulargelenkes.

eine Subluxationsstellung hin. Die Verletzung des SC-Gelenkes kann insbesondere bei Polytraumatisierten übersehen werden.

Im Falle eines SCG-Luxationsverdachtes kommt als erster Schritt der bildgebenden Verfahren eine Röntgen-Nativ-Aufnahme beider Sternoklavikulargelenke und des Thorax infrage. Eine SpezialRöntgenaufnahme kann mit Hilfe der Technik nach Rockwood mit einer 40 Grad nach kranial gekippten Röhre erfolgen (Abb.8). Die klinisch und radiologisch sichtbare Asymmetrie führt $\mathrm{zu}$ einer CT-Untersuchung. Mit einer horizontalen Rekonstruktion gelingt der Nachweis einer vorderen oder hinteren Luxation [7,13,14], (Abb.9).

Die ACG-Verletzung ist durch die hochstehende, federnde Klavikula (Klaviertastenphänomen) und die druckschmerzhafte Schwellung im Seitvergleich leicht erkennbar.

Eine röntgenologische a.-p.-Darstellung der Schultergelenke im Sinne einer Panorama-Aufnahme, bei eindeutiger Klinik gleich als Panorama-Belastungsaufnahme mit am Handgelenk hängenden Gewichten von 5 oder $10 \mathrm{~kg}$, kann als Standard bezeichnet werden (Abb.13-16). Die Zielaufnahme des AC-Gelenkes, zur Beurteilung der Gelenkspaltweite bzw. arthrotischen Veränderungen, erfolgt mit um 20 Grad nach kranial gekippter Röhre $[7,9,12]$.

Die Diagnose einer SCG- oder ACGSprengung ist eine klinische Diagnose. Der goldene Standard ist in der Diagnostik einer SCG-Luxation die CT-Untersuchung und im Falle einer ACG-Luxation die Panorama-Belastungsaufnahme.

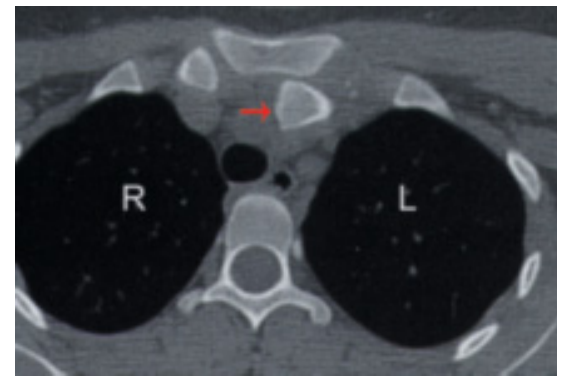

Abb. 9 Dorsale Luxation des SCG links (Pfeil), rechts regelrechte Gelenkstellung.

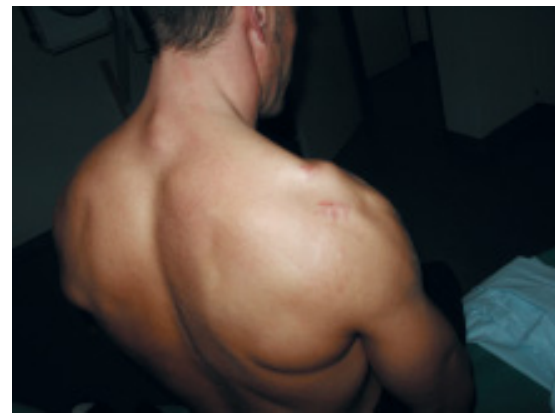

Abb.10 Direkter Sturz auf die rechte Schulter, Schürfung der Haut und Hochstand der Klavikula.

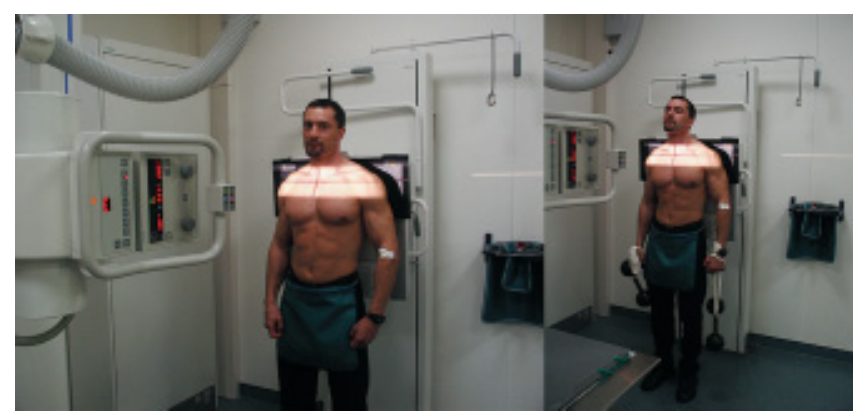

13

14

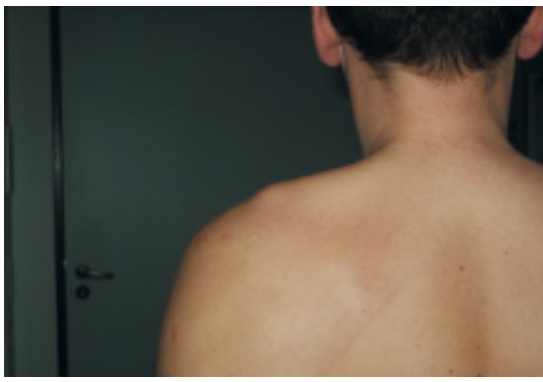

Abb.11 Links: Klavikulahochstand.

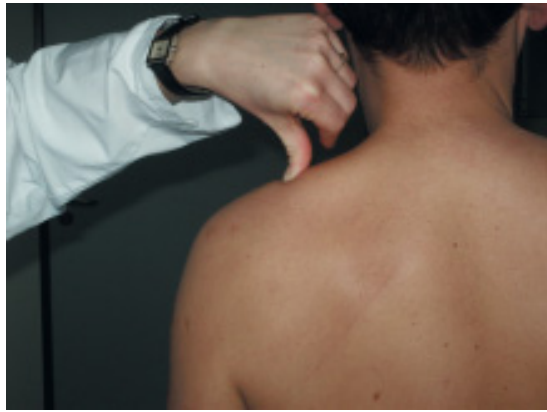

Abb.12 Rechts: Klaviertastenphänomen.

Abb.13 Links: Panorama-Aufnahme.

Abb. 14 Rechts: Mit $5 \mathrm{~kg}$ Belastung bds. 


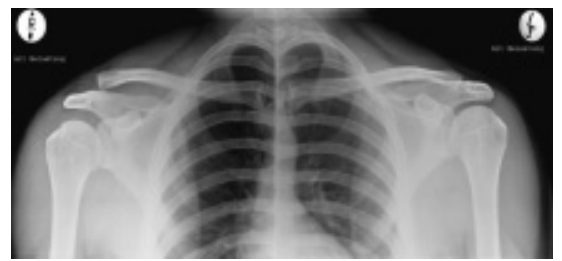

Abb.15 Regelrechte Artikulation im ACG links, rechts Nachweis einer Sprengung mit Hochstand der Klavikula um eine Schaftbreite und Verdoppelung der korakoklavikulären Distanz.

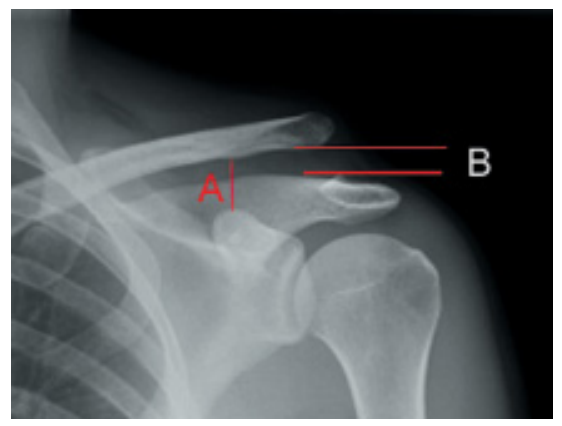

Abb.16 Beurteilung der AC-Gelenkmaße auf der Basis der Belastungsaufnahme. (a) Distanz Korakoid-Klavikula $>=14 \mathrm{~mm}$. (b) Akromioklavikuläre Stufe $>=8 \mathrm{~mm}$.

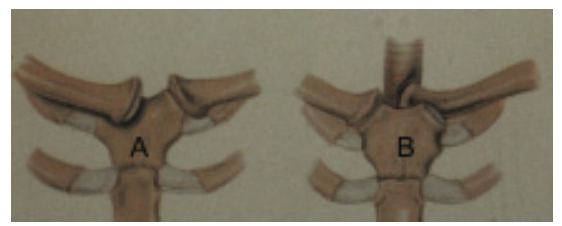

Abb.17 (A) Vordere Luxation des SCG, (B) hintere Luxation.

\section{Klassifikationen}

Nach Allmann werden am SCG 3 Verletzungstypen unterschieden:

Typ I: Zerrung der Gelenkkapsel und der sternoklavikulären Bänder

Typ II: Ruptur der Gelenkkapsel und der sternoklavikulären Bänder, kostoklavikuläre Bänder noch intakt, wodurch eine Subluxation des Gelenkes entsteht

Typ III: Ruptur der Gelenkkapsel und der sternoklavikulären Bänder und Durchtrennung der Bandverbindung der Klavikula zur ersten Rippe

Die Typ-III-Verletzung wird je nach Richtung der Gewalteinwirkung in eine vordere (indirekte Gewalteinwirkung) und eine hintere Luxation (direkte Gewalteinwirkung auf die mediale Klavikula) eingeteilt (Abb. 17a u. b).

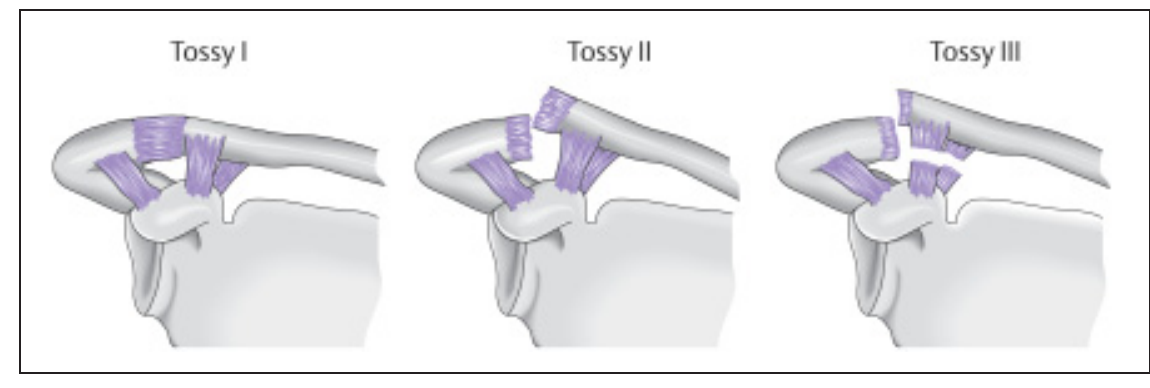

Abb.18 Tossy-Klassifikation der AC-Gelenkverletzung.

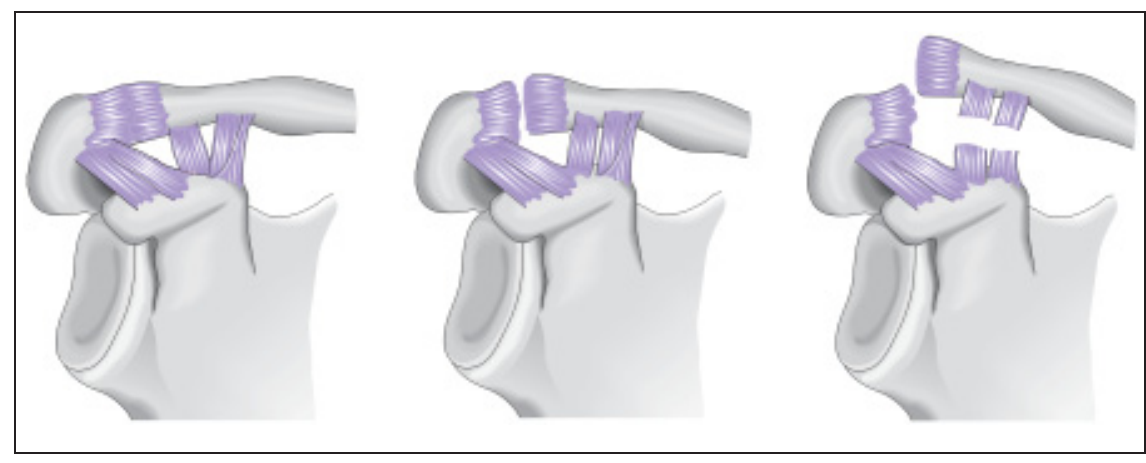

Von links nach rechts Rockwood I-III

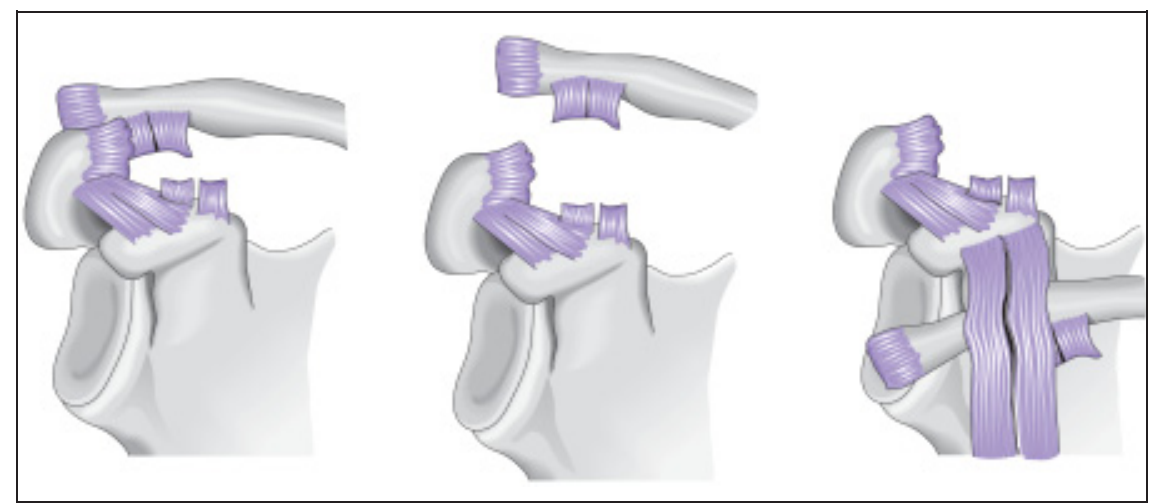

Von links nach rechts Rockwood IV - VI

Abb.19 Klassifikation nach Rockwood.

\section{Klassifikation der Akromioklavikulargelenksprengung nach Tossy}

Typ I: Zerrung des Lig. acromioclaviculare, Lig. Korakoklavikulare bleibt intakt (Abb. 20/20a)

Typ II: Ruptur des Lig. acromioclaviculare, Teilruptur des Lig. coracoclaviculare und Subluxation im ACG (Abb.21/21 a)

Typ III: Ruptur des Lig. acromioclaviculare und coracoclaviculare, Luxation des Gelenkes [10,16] (Abb. 22)
Bei der ACG-Verletzung wird die TossyKlassifikation zunehmend durch die Klassifikation nach Rockwood ersetzt.

\section{Klassifikation der ACG-Sprengung nach Rockwood}

Typ I: Zerrung des Lig. acromioclaviculare, Lig. coracoclaviculare bleibt intakt

Typ II: Ruptur des Lig. acromioclaviculare, Subluxation im ACG

Typ III: Ruptur des Lig. acromioclaviculare und coracoclaviculare, Luxation des Gelenkes, korakoklavikulärer Abstand um $30-100 \%$ vergrößert 


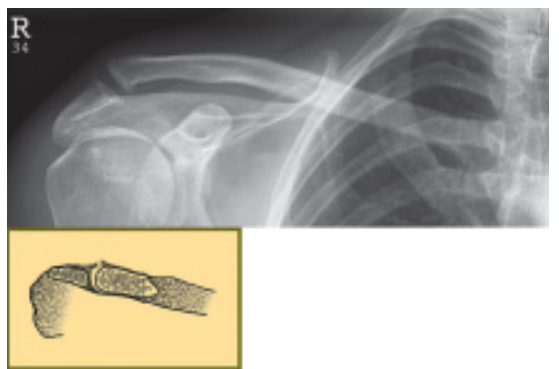

Abb. 20/20a Normale Röntgen-Anatomie einer Tossy-I/Rockwood-I-Verletzung.

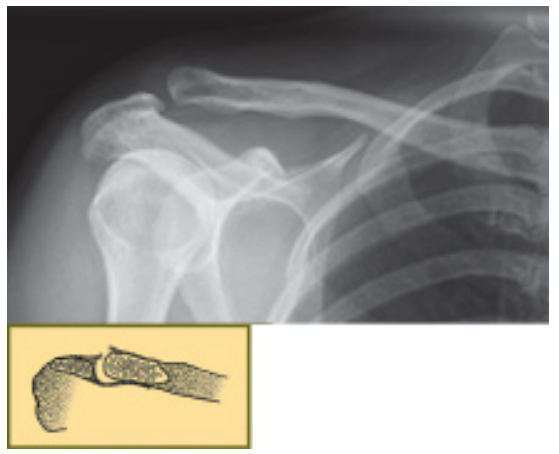

Abb.21/21 a Röntgen-Aufnahme einer TypII-Verletzung (Rockwood/Tossy) mit Subluxationsstellung im ACG und Hochstand der Klavikula um $1 / 2$ Schaftbreite.

Typ IV: Dorsale Luxation der Klavikula im ACG

Typ V: Ruptur des Lig. acromioclaviculare und coracoclaviculare, Luxation des Gelenkes, korakoklavikulärer Abstand mehr als $100 \%$ vergrößert

Typ VI: Luxation nach kaudal unter das Akromion [10,12,13].

Die SCG-Verletzung wird nach Allmann und die ACG-Verletzung nach Rockwood eingeteilt.

\section{Therapie SC-Gelenkverletzung}

Distorsionen und Subluxationen des SCG werden konservativ behandelt. Kurzfristige schmerzabhängige Ruhigstellung in einer Armschlinge, Eisapplikation und antiphlogistische Behandlung sind allgemein akzeptierte Maßnahmen.

Bei den Luxationen, Typ-III-Verletzung nach Allmann, muss ein geschlossener Repositionsversuch unternommen werden.

Der 90 Grad abduzierte Arm wird in Verlängerung der Klavikula gezogen, bei der ventralen Luxation wird auf das mediale

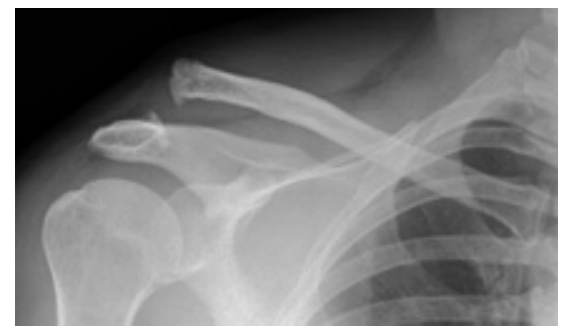

Abb. 22 Typ-V-Verletzung nach RockwoodKlassifikation mit Luxation des Gelenkes, korakoklavikulärer Abstand meht als $100 \%$ vergröBert.

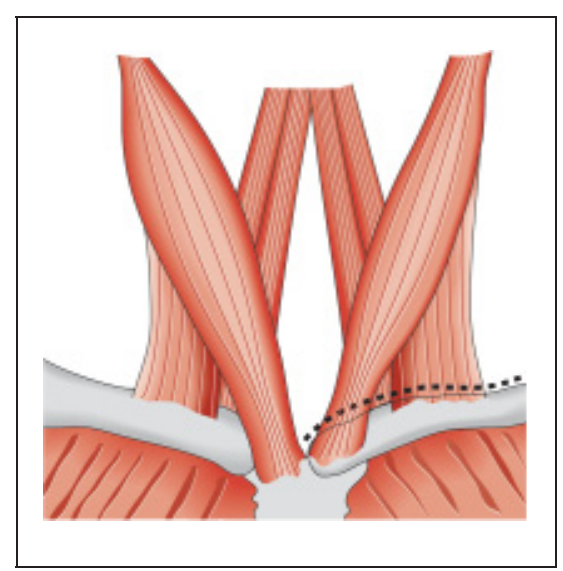

Abb. 23 Zugang zum SC-Gelenk.

klavikularende Druck ausgeübt. Im Falle einer hinteren Luxation muss die Klavikula hinter dem Manubrium sterni hervorgeholt werden. Unter Umständen muss beim Repositionsmanöver die Klavikula mit einer Repositionszange gefasst werden. Die hintere Luxation im SCG kann zu schweren retrosternalen Begleitverletzungen, z.B. der Gefäße, Trachea oder Pleura führen, aus diesem Grund muss eine geschlossene Reposition so schnell wie möglich erfolgen. Die postoperative Ruhigstellung erfolgt nach ventraler Luxation in einem RucksackVerband; nach einer dorsalen SCG-Luxation im Gilchrist-Verband. Das Gelenk sollte insgesamt 6 Wochen ruhig gestellt werden.

Wird nach geschlossener Reposition keine Stabilität und sichere Retention erreicht ist die offene Reposition und Stabilisierung indiziert.

Die Retention im SCG wird bei den unterschiedlichen OP-Verfahern entweder mit 8-förmiger doppelter Drahtcerclage, retrosternaler Balser-Platte, Cerclage mittels PDS-Kordel oder der Sehne des M. palmaris longus erreicht $[3,6,14]$.

\section{Operationstechnik}

Achterförmige doppelte Drahtcerclage über das SCG

\section{Vorgehen der Autoren}

Lagerung des Patienten in halbsitzender Position, bogenförmige Hautinszision über das SCG vom medialen Klavikuladrittel bis zum Manubrium sterni (Abb. 23). Mobilisation der Haut und des subkutanen Fettgewebes, Beginn dieser Präparation von der Klavikula aus. Darstellung der Gelenkkapsel und der sternoklavikulären Bänder, Beurteilung des Discus artikularis ggf. Entfernen desselben. Zur Darstellung der kostoklavikulären Bänder muss der M. pectoralis nach kaudal abgelöst werden. Nach der Reposition werden die Nähte für die Bänder und Gelenkkapsel mit resorbierbarem Nahtmaterial Vicryl der Stärke 2/0 vorgelegt. Die doppelte Drahtcerclage $(1,25 \mathrm{~mm}$ Draht) wird nach Anlegen von V-förmigen Bohrkanälen am Manubrium sterni unter Schonung der dorsalen Wand und ein queres Bohrloch an der Klavikula (Bohrer Stärke 3,2 mm) angefertigt (Abb. 24a-c).

Die postoperative Nachbehandlung besteht aus einer 4-wöchigen Ruhigstellung im Gilchrist-Verband oder Ultra-Sling-IIVerband, nach Ablauf der 4. Woche wird die Ruhigstellung für weitere 2 Wochen mit Armschlinge durchgeführt, gleichzeitig wird mit passiven und aktiven Bewegungsübungen des Schultergelenkes begonnen. Mit der Belastung des Armes, sportlicher Aktivität kann nach Ablauf des 4. postoperativen Monats begonnen werden.

\section{Komolikationen}

Auf die hohe Rate der Reluxationen nach einer operativen Stabilisierung, die kosmetisch störende Narbe und in Anbetracht der Nähe des Mediastinums die gefährliche Materialdislokation (vor allem Kirschner-Drähte), wird in allen Veröffentlichungen hingewiesen $[1,3,6,10]$.

\section{Therapie der chronischen Instabilität des Sternoklavilkulargelenkes}

Die schmerzbedingte Bewegungseinschränkung im Schultergelenk bei chronischer Instabilität des SCG stellt eine Operationsindikation dar. Zusätzlich ist der Patient durch die sichtbare Asymmetrie bei vorstehendem Klavikulaende belastet. 
a, b
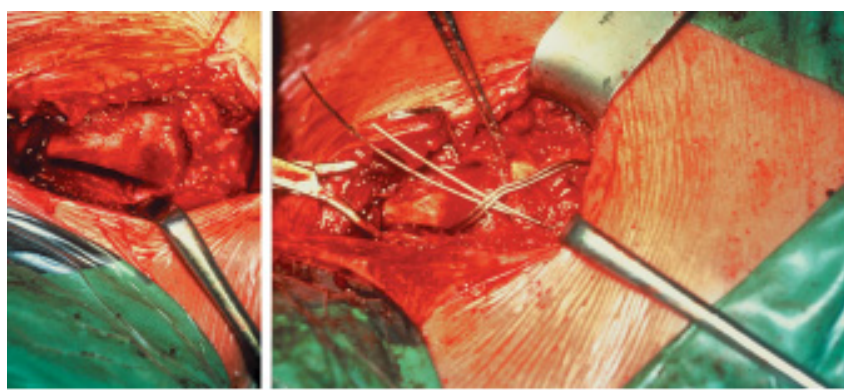

c, d
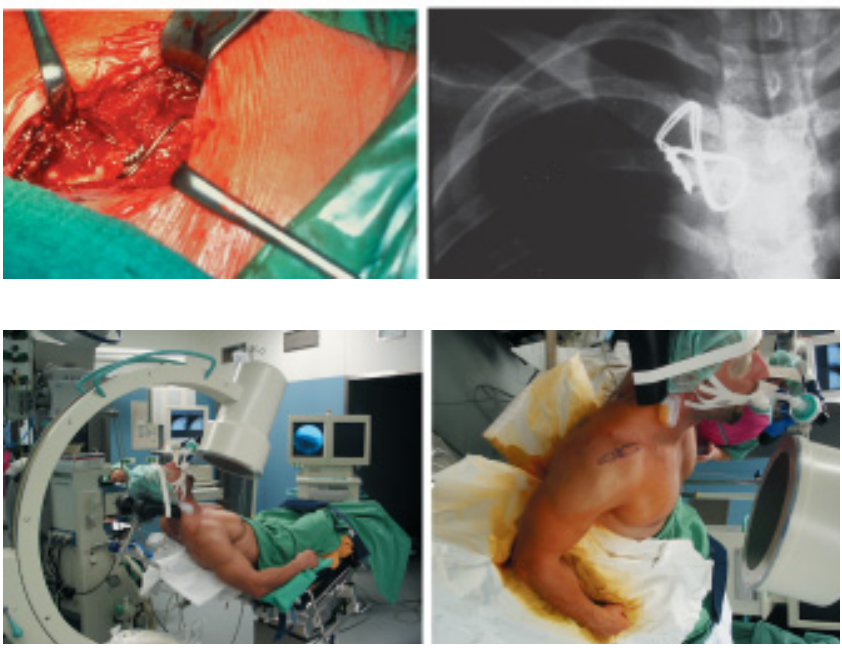

Mit der Resektionsarthroplastik kann bei veraltetem bzw. degenerativ verändertem Sternoklavikulargelenk ein gutes Ergebnis erzielt werden.

Der Patient wird in halbsitzender Position gelagert, bogenförmige Hautinszision über dem SCG vom medialen Klavikuladrittel bis zum Manubrium sterni. Mobilisation der Haut und des subkutanen Fettgewebes. Resektion der Gelenkkapsel, der sternoklavikulären Bänder und des Discus articularis. Nach Ablösen des M. sternocleidomastoideus erfolgt die Klavikularesektion medial der sternoklavikulären Bänder.

Distorsionen und Subluxationen des SCG werden konservativ behandelt. Werden nach geschlossener Reposition einer SCG-Luxation keine Stabilität und sichere Retention erreicht, so ist die offene Reposition und Stabilisierung indiziert.

\section{Therapie der AC-Gelenkverletzung}

Die Verletzungen vom Typ Tossy I+ II/ Rockwood I + II werden konservativ behandelt.

Die Behandlung besteht in kurzzeitiger Ruhigstellung im Gilchrist- oder UltraSling-Verband, alternativ kann die betrof-
Abb. 24 (a-c) Darstellung des SCG, Reposition der hinteren Luxation, Anfertigung doppelter Cerclage mit 1,25 mm-Draht, postoperative Röntgen-Kontrolle.

Abb. 25 (a u. b) Lagerung in halbsitzender Beach-chairPosition.
Zum Beispiel ergab die Metaanalyse von 600 Veröffentlichungen zwischen 1966 und 1997 in den amerikanischen Journals mit einer Patientenzahl von 1172, darunter 833 operative Fälle, gute bis sehr gute Ergebnisse bei $88 \%$ der operativ Behandelten und 87\% der konservativ Behandelten. Unter diesen 600 Veröffentlichungen waren lediglich 4 Studien mit prospektiv randomisiertem Design und einer Patientenzahl über 100.

Eine Umfrage an unfallchirurgischen Kliniken in Deutschland zeigte folgendes Ergebnis: 63\% der Befragten meinten, dass das Ergebnis der operativen Therapie der konservativen überlegen sei. Von den restlichen 37\% der Kliniken, die eine konservative Therapie mindestens als gleichwertig einschätzen, führten nach ihren eigenen Angaben lediglich 13\% diese auch tatsächlich bei Tossy- bzw. Rockwood III-Verletzung durch $[2,4,5,7,9,11]$.

Die Vielzahl der Operationstechniken lässt sich am besten anhand des verwendeten Mateials in drei Gruppen einteilen. Die Reposition im Akromioklavikulargelenk wird bei den unterschiedlichen Verfahren entweder mit resorbierbaren, nicht resorbierbaren Materialien oder aber mit einer Kombination aus beiden erreicht. gestellt werden. Die Dauer der Immobilisation wird von der Schmerzsymptomatik der Patienten bestimmt, allerdings nicht länger als 7-10 Tage. Begleitend sollten die Kühlung und die antiphlogistische Behandlung durchgeführt werden. Bei der Typ-I-Verletzung kann nach der Schmerzfreiheit mit körperlicher/sportlicher Belastung begonnen werden. Bei der Verletzung des ACG von Typ II sollte die körperliche bzw. sportliche Belastung für die Dauer von 4 Wochen vermieden werden.

Die Verletzungen vom Typ Rockwood IV VI werden operativ versorgt.

Die Behandlung der III-gradigen Verletzung des ACG bleibt ein aktuelles Diskussionsthema. Es besteht keine Einigkeit über die Wahl des Therapieverfahrens. Den Hauptgrund hierfür sehen wir im Fehlen prospektiv randomisierter Studien.

In zahlreichen Veröffentlichungen zeigten sich vergleichbar gute bis sehr gute Ergebnisse der konservativen und der operativen Therapie der Tossy- bzw. Rockwood-III-Verletzung des Schultereckgelenkes.

Aufrechterhaltung der Reposition nötige Material durch das ACG positioniert. Die temporäre Transfixation sei als Beispiel dafür genannt.

Bei extraartikulärer Fixation wird entweder das ACG überbrückt - Beispiel für diese Art der Fixierung sind die verschiedenen Platten - oder das Material liegt außerhalb des Gelenkes. In diese Gruppe können Verfahren, bei denen die Reposi- 

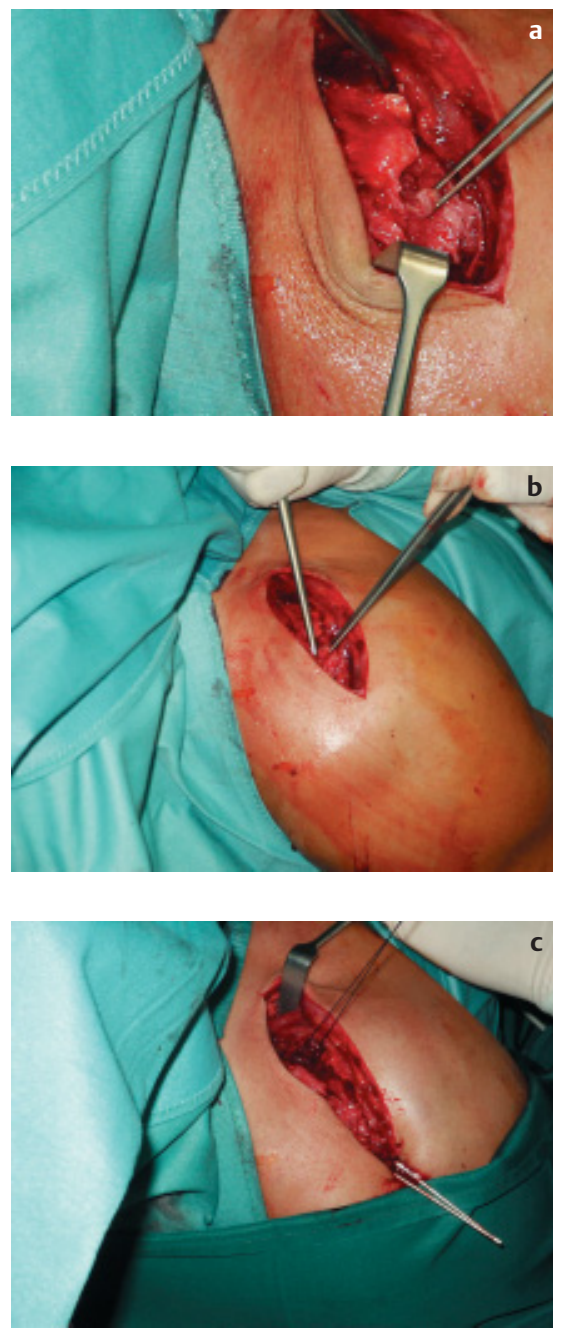

tion durch korakoklavikuläre Augmentation oder durch die Verwendung der korakoklavikulären Schraube (z.B. die Schraube nach Bosworth) aufrechterhalten wird, eingeordnet werden $[2,4,5,11$, $12,15]$.

Die AC-Gelenkverletzungen vom Typ Rockwood I+ II werden konservativ behandelt. Die Behandlung der ACG-Luxation wird nach wie vor kontrovers diskutiert, die Mehrzahl der Unfallverletzten mit einer Luxation wird an unfallchirurgischen Kliniken Deutschlands operativ behandelt.

\section{OP-Technik}

Transartikuläre Kirschner-Drähte mit Zuggurtungscerclage

(Vorgehen der Autoren)

Die Operation erfolgt in Allgemeinnarkose, der Patient wird in halbsitzender Position gelagert (Beach-chair-Position).
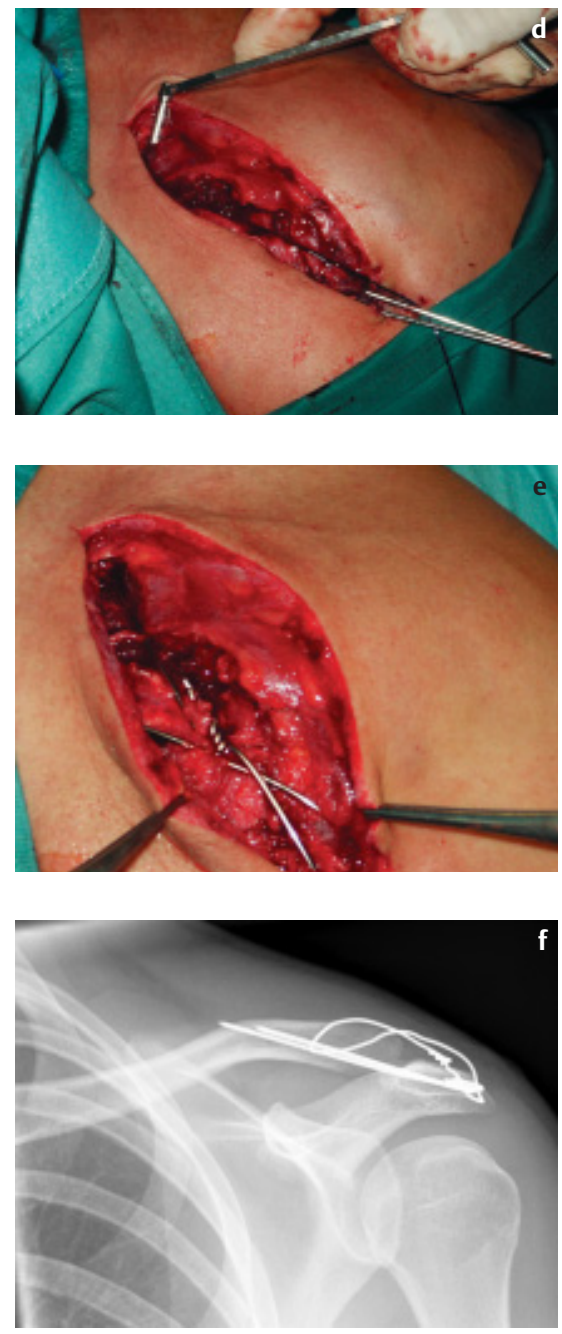

Der Arm wird frei beweglich abgedeckt, die betreffende Schulter und der proximale Teil des Armes bleiben frei. Der Zugang zum Akromioklavikulargelenk erfolgt durch einen infraklavikulären Hautschnitt, der lateral des AC-Gelenkes nach dorsal umbiegt (Hockey-SchlägerSchnitt). Das Schultereckgelenk und das laterale Ende der Klavikula werden dargestellt und der Zustand der akromioklavikularen Bänder, des Gelenkes und des Diskus beurteilt. Der eventuell vorhandene Discus articularis wird begutachtet und möglichst - abhängig vom Grad der Schädigung - erhalten. Die Nähte für den Bandapparat zwischen Korakoid und Klavikula mit 2/0 Vicrylnähten werden zuerst nur vorgelegt. Durch die laterale Klavikula wird ein $2 \mathrm{~mm}$ Bohrkanal angelegt, durch den der 1,25 mm Zuggurtungsdraht gezogen wird. Das Akromion ist ausreichend freigelegt. Dann erfolgt die anatomische Reposition des Akromioklavikulargelenkes und das Einschießen von 2 jeweils $1,8 \mathrm{~mm}$ starken KirschnerDrähten transartikulär durch das Akro-

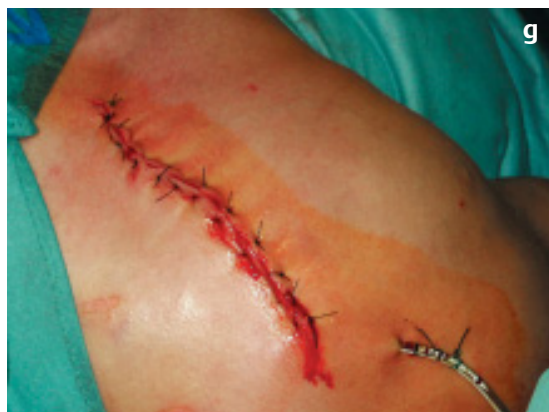

Abb.26 (a) Darstellung des Gelenkes, Beurteilung der Bänder, des Diskus. (b) Repostion. (c) Vorgelegte Nähte für den Bandapparat, CCBandkomplex, nach anatomischer Reposition Transfixation des Gelenkes mit 2 1,8 mm starken Kirschner-Drähten. (d, e) Anlagen eines $2 \mathrm{~mm}$ Bohrkanals, durch den ein $1,25 \mathrm{~mm}$ Zuggurtungsdraht gezogen wird, achterförmiger Zuggurtungsdraht wird leicht angezogen und verzwirbelt. (f) Die Drahtenden sind umgebogen und im Akromion versenkt. (g) Drainage und Naht. mion in die laterale Klavikula. Die korrekte Position wird durch BV-Kontrolle geprüft. Der Zuggurtungsdraht wird achterförmig um die Drahtenden gelegt, nicht zu sehr angezogen und verzwirbelt. Dann werden die Drahtenden umgebogen und im Akromion versenkt. Die vorgelegten Nähte des Bandapparates werden geknotet. Mit Hilfe des Bildwandlers wird nochmals die korrekte Reposition des Akromioklavikulargelenkes und die Lage des Implantates überprüft. Auf die anatomische Weite des Gelenkes muss geachtet werden. Es erfolgt die Spülung und das Einlegen einer Drainage, und schichtweise der Wundverschluss.

Postoperativ wird der Arm auf Kissen gelagert. Nach dem Entfernen des Nahtmaterials wird der Verband, z. B. Ultra Sling II oder Gilchrist-Verband, durch eine Armschlinge ersetzt. Die Metallentfernung erfolgt nach 6 Wochen. Bis zu diesem Zeitpunkt ist das Abspreizen und Anheben des Armes über die Horizontale zu vermeiden (Abb. 26a-g). 


\section{Therapie der chronischen Instabilität des Akromioklavikulargelenkes}

Die laterale Klavikularesektion ist das Verfahren der Wahl bei chronischer Instabilität, veralteter Luxation des AC-Gelenkes und bei Sprengungen des Gelenkes mit Trümmerfraktur des lateralen Klavikulaendes. Die erste laterale Klavikularesektion führte Fracassini 1902 durch. Gurd und Mumford veröffentlichten 1941 die o.g. Indikationen für dieses OP-Verfahren.

Weaver und Dunn kombinierten die laterale Resektion der Klavikula mit Ligamentoplastik, nach Resektion von $2 \mathrm{~cm}$ der distalen Klavikula wird korakoklavikuläres Ligament am Klavikulaende transferiert.

\section{Schlussfolgerung}

Die Diagnose einer SCG- oder ACG-Sprengung ist eine klinische Diagnose. Der goldene Standard ist in der Diagnostik einer SCG-Luxation die CT-Untersuchung und im Falle einer ACG-Luxation die Panorama-Belastungsaufnahme.

Die SCG-Verletzung wird nach Allmann und die ACG-Verletzung nach Rockwood/Tossy eingeteilt.

Distorsionen und Subluxationen des SCG werden konservativ behandelt. Wird nach geschlossener Reposition einer SCG-Luxation keine Stabilität und sichere Retention erreicht, so ist die offene Reposition und Stabilisierung indiziert.

Die AC-Gelenkverletzungen vom Typ Tossy I + II/Rockwood I+ II werden konservativ behandelt. Die Behandlung der ACG-Luxation wird nach wie vor kontrovers diskutiert, die Mehrzahl der Unfallverletzten mit einer Luxation wird an unfallchirurgischen Kliniken Deutschlands operativ behandelt.

\section{Literatur}

${ }^{1}$ Allmann Fl. Fractures and Ligamentous Injuries of the Clavicle and its Articulation. J Bone Jt Surg Am 1967; 49: 774-784

${ }^{2}$ Bäthis H, Tingart M, Bouillon B. Stand der Therapie der Schultereckverletzung. Unfallchirurg 2001; 104: 955-960

${ }^{3}$ Franck WM, Müller O, Hennig F. Die traumatische sternoklavikuläre Instabilität. Unfallchirurg 2000; 103: 834-838

${ }^{4}$ Galpin RD, Hawkins. A comparative analysis of operative vs. nonoperative Treatment of grade III acromioclavicular Separation. Clin Orthop 1985; 193: 151 - 155

${ }^{5}$ Göhring U. Behandlungsergebnisse nach unterschiedlichen Operationsverfahren zur Versorgung einer Schultereckgelenksprengung. Chirurg 1993; 64: 565-571

${ }^{6}$ Haas N, Blauth M. Verletzungen des Acromio- und Sternoclaviculargelenkes. Operative und konservative Behandlung. Orthopäde 1989; 18: 234-247

7 Jerosch J. Das Akromioklavikulargelenk. Orthopäde 2000; 29: 895-908

${ }^{8}$ Karpandji IA. Funktionelle Anatomie der Gelenke. I. Obere Extremität, F. Enke, Stuttgart 1984

${ }^{9}$ Mayr E, Braun W, Eber W. Versorgung von ACG-Sprengungen. Unfallchirurg 1999; 102: $278-286$

${ }^{10}$ Mutschler W, Haas NP. Praxis der Unfallchirurgie. Georg Thieme Verlag, Stuttgart, New York 1999

${ }^{11}$ Nachtkamp J, Magin J, Paar O. Die operative Behandlung der ACG-Sprengung. Akt Traumatol 1996; 26: $42-47$

${ }^{12}$ Phillips AM, Smart C, Groom AFG. Acromioclavicular Dislocation. Clinic Orthop Relat Res 1998; 535: 10-17

${ }^{13}$ Rockwood CA Jr, Green DP. Fractures in Adult. Injuries to the Acromioclavicular Joint, Lippincott, Philadelphia 1984, S. 960-982

${ }^{14}$ Rowe CR. Acromioclavicular and Sternoclavicular Joints. The Shoulder, Churchill Livingstone, New York, London, Melbourne 1988, S. 293-315

${ }^{15}$ Thelen E, Rehn J. Acromioclavicularsprengungen, Ergebnisse nach operativer und konservativer Versorgung in 162 Fällen. Unfallheilkunde 1976; 79: 417-422

${ }^{16}$ Tossy JD, Newton CM, Sigmond HM. Acromioclavicular separations. Useful and practical classification for treatment. Clin Orthop 1963; 28: 111 - 119

\section{Dr. med. Dara Orangi \\ Oberarzt}

Prof. Dr. med. Karl Heinrich Winker

Chefarzt

Klinik für Unfall-, Hand- und Wiederherstellungschirurgie HELIOS Klinikum Erfurt

Nordhäuserstr. 74

D-99089 Erfurt 